15 CARDIAC MAGNETIC RESONANCE (CMR) ASSESSMENT OF RIGHT VENTRICULAR-PULMONARY ARTERIAL COUPLING AND RIGHT VENTRICULAR TRABECULAR COMPLEXITY: IMPACT ON PROGNOSIS IN PATIENTS UNDERGOING LUNG TRANSPLANT ASSESSMENT

G Sarri, K Halim, M Mccurry, I Pierce, S Rahman-Haley, A Yesudass, M Joseph, A Simon, M Carby, A Reed, J Wong. Harefield Hospital, Royal Brompton and Harefield Foundation Trust, Uxbridge, UK

10.1136/heartjnl-2019-BSCMR.15

Introduction Right ventricular (RV) function strongly influences prognosis in pulmonary hypertension (PH), but it remains

Abstract 15 Table 1 CMR and echo variables, correlations and outcomes in patients undergoing lung transplant assessment

\begin{tabular}{|c|c|c|c|c|c|c|c|c|c|c|}
\hline & \multicolumn{2}{|c|}{ All patients $(n=84)$} & \multicolumn{2}{|c|}{ Alive $(n=70)$} & \multicolumn{2}{|c|}{ Dead $(n=14)$} & \multirow{2}{*}{$\begin{array}{l}\text { Alive vs dead } \\
\mathrm{p} \text { value }\end{array}$} & \multirow[t]{2}{*}{ HR } & \multirow[t]{2}{*}{$\mathrm{Cl}$} & \multirow[t]{2}{*}{$p$ value } \\
\hline & $\begin{array}{l}\text { Mean/ } \\
\text { median }\end{array}$ & SEM/IQR & $\begin{array}{l}\text { Mean/ } \\
\text { median }\end{array}$ & SEM/IQR & $\begin{array}{l}\text { Mean/ } \\
\text { median }\end{array}$ & SEM/IQR & & & & \\
\hline \multicolumn{11}{|l|}{ Demographics } \\
\hline Age (years) & 53 & 16 & 53 & 15 & 50 & 18 & 0,26 & & & \\
\hline Gender (M) & 46 & & 37 & & 9 & & 0,43 & & & \\
\hline BSA & 1,78 & 0,02 & 1,78 & 0,03 & 1,79 & 0,05 & 0,84 & & & \\
\hline Ethnicity & & & & & & & overall 0.90 & & & \\
\hline White & 72 & & 60 & & 12 & & & & & \\
\hline African & 2 & & 2 & & 0 & & & & & \\
\hline Afro-Caribbean & 6 & & 5 & & 1 & & & & & \\
\hline Asian & 4 & & 3 & & 1 & & & & & \\
\hline \multicolumn{11}{|l|}{ Diagnosis } \\
\hline ILD & 28 & & 23 & & 5 & & & & & \\
\hline Emphysema & 32 & & 27 & & 5 & & & & & \\
\hline Cystic Fibrosis & 15 & & 14 & & 1 & & & & & \\
\hline Bronchiectasis & 5 & & 3 & & 2 & & & & & \\
\hline Other & 3 & & 2 & & 1 & & & & & \\
\hline $\begin{array}{l}\text { Pulmonary hypertension (echo } \\
\text { diagnosis, } n=51 \text { ) }\end{array}$ & 51 & & 41 & & 10 & & 0,58 & & & \\
\hline $\begin{array}{l}\text { Clinical classification of patients } \\
\text { with PH ( } n=51)\end{array}$ & & & & & & & overall 0.64 & & & \\
\hline Group 1 & 3 & & 3 & & 0 & & & & & \\
\hline Group 3 & 40 & & 32 & & 8 & & & & & \\
\hline Group 5 & 8 & & 6 & & 2 & & & & & \\
\hline Transplanted & 22 & & 15 & & 7 & & 0,03 & 2,06 & $0.68-6.22$ & 0,20 \\
\hline \multicolumn{11}{|l|}{ MRI and Echo indices } \\
\hline CMR LVESVI & 22 & 13 & 21 & 13 & 25 & 13 & 0,30 & & & \\
\hline CMR LV StVI & 38 & 1,54 & 37 & 1,43 & 39 & 6,04 & 0,63 & & & \\
\hline CMR LVEF & 62 & 1,07 & 63 & 1,14 & 58 & 2,77 & 0,06 & & & \\
\hline CMR RVEDVI & 72 & 32 & 70 & 28 & 83 & 62 & 0,01 & 1,03 & $1.01-1.04$ & $<0.005$ \\
\hline CMR RVESVI & 41 & 2,62 & 37 & 2,06 & 64 & 10,14 & 0,02 & 1,03 & $1.01-1.04$ & $<0.005$ \\
\hline CMR RV StVI & 37 & 14 & 37 & 13 & 35 & 11 & 0,49 & & & \\
\hline CMR RVEF & 51 & 18 & 53 & 15 & 38 & 17 & ,001 & 0,94 & $0.90-0.97$ & $<0.005$ \\
\hline CMR 4ch RAArea & 16 & 7 & 16 & 6 & 20 & 6 & 0,11 & & & \\
\hline LGE & 14 & & 9 & & 5 & & 0,04 & 2,45 & $0.79-7.61$ & 0,12 \\
\hline Non-insertion point LGE & 7 & & 5 & & 2 & & 0,38 & & & \\
\hline RV-PA coupling SV/ESV & 1,03 & 0,72 & 1,12 & 0,67 & 0,57 & 0,48 & ,001 & 0,12 & $0.027-0.52$ & $<0.005$ \\
\hline Global FD & 1,26 &, 004 & 1,26 &, 004 & 1,26 &, 012 & 0,49 & & & \\
\hline Maximal Basal FD & 1,33 &, 007 & 1,32 &, 008 & 1,33 &, 017 & 0,35 & & & \\
\hline Mean Basal FD & 1,26 &, 006 & 1,25 &, 007 & 1,28 &, 014 & 0,06 & & & \\
\hline Maximal Apical FD & 1,30 &, 007 & 1,30 &, 008 & 1,30 &, 016 & 0,74 & & & \\
\hline Mean Apical FD & 1,25 &, 007 & 1,25 &, 007 & 1,25 &, 016 & 0,80 & & & \\
\hline
\end{tabular}




\begin{tabular}{|c|c|c|c|c|c|c|}
\hline \multicolumn{7}{|c|}{ Correlations } \\
\hline & RV EDVI & RV ESVI & RV SVI & RV EF & RA area & mPAP \\
\hline \multicolumn{7}{|l|}{ SV/ESV } \\
\hline$r$ value & $-0,407$ & $-0,712$ & 0250 & 0847 & $-0,231$ & $-0,301$ \\
\hline$p$ value & $<0.001$ & $<0.001$ &, 022 & $<0.001$ &, 042 & ,014 \\
\hline \multicolumn{7}{|c|}{ Global FD } \\
\hline$r$ value & 319 & ,303 & 130 &,- 203 & ,280 & 290 \\
\hline$p$ value & $<0.005$ &, 005 & 238 &, 064 & ,013 & 018 \\
\hline \multicolumn{7}{|c|}{ Maximal Basal FD } \\
\hline$r$ value & ,389 & ,350 & ,226 & -196 & ,296 & 267 \\
\hline$p$ value & $<0.001$ & $<0.005$ & 039 &, 073 &, 008 &, 030 \\
\hline \multicolumn{7}{|c|}{ Mean Basal FD } \\
\hline$r$ value & ,401 & ,373 & , 179 &,- 215 &, 350 & ,297 \\
\hline$p$ value & $<0.001$ & $<0.001$ & 102 &, 050 & $<0.005$ & 016 \\
\hline
\end{tabular}

BSA: body surface area; ILD: Interstitial lung disease; LVEDVi or RVEDVi: indexed left ventricular or right ventricular end-diastolic volume; LVESVi or RVESVi: indexed LV or RV end systolic volume; LV StVI or RV StVI: indexed LV or RV stroke volume; LVEF or RVEF: LV or RV ejection fraction; LGE: late gadolinium enhancement; FD: fractal dimension; HR: hazard ratio; Cl: confidence interval, SEM/IQR: standard error of mean/interquartile range )

unclear what key metrics are most clinically relevant. The purpose of this study was to assess the clinical relevance of both RV trabecular complexity and adequacy of RV functional adaptation to increased afterload as assessed by CMR in patients undergoing lung transplant assessment.

Methods Between 2013 and 2018, 84 consecutive patients underwent lung transplant assessment with echocardiography and CMR (1.5T - Siemens Aera) to assess biventricular volumes and function as well as late gadolinium enhancement (LGE). RV trabecular complexity was assessed by its fractal dimension (FD) on CMR, using freely available code (FracAnalyse). RV functional adaptation to increased afterload was assessed with the RV-pulmonary arterial (PA) coupling index (stroke volume(SV)/end-systolic volume(ESV) ratio). Survival was analyzed using the Cox proportional hazard ratio with the primary outcome of time to death.

Results In total 84 patients (median age 53 \pm 16 years, 54\% male) were included in analysis. Median follow up period was $19.3 \pm 17.2$ months. Underlying lung disease was recorded in $98 \%$ of the study population. Tricuspid regurgitation was echo-detected in 66 patients; 77\% (51pts) had echo-calculated pulmonary hypertension $(\mathrm{PH})$ with an estimated RV systolic pressure $>35 \mathrm{mmHg}$ and estimated mean PA pressure of 30 $\pm 10 \mathrm{mmHg}$. $78.4 \%, 15.7 \%$, and $5.9 \%$ of $\mathrm{PH}$ patients were categorized to Groups 3, 5 and 1 of WHO PH classification respectively. Mean LV and RV ejection fraction were $62 \%$ $\pm 1.07 \%$ and $51 \% \pm 18 \%$ respectively.

Both SV/ESV and RV FD correlated to mPAP, CMR-derived right atrial area, RVEDVi and RVESVi (table 1). RV FD did not differ significantly in patients with PH. Survival was predicted by SV/ESV ratio, RVEF, indexed RV end-diastolic volume (EDVi), RV end-systolic volume (ESVi), and mPAP on univariate analysis (table 1 ; SV/ESV carried a hazard ratio of $0.12, \mathrm{p}<0.005)$.

Conclusions In patients with underlying lung disease referred for lung transplant assessment, RV functional adaptation to afterload assessed by CMR seems to predict survival. Fractal analysis of RV trabecular complexity correlated with metrics influencing RV remodelling and contractility, although not survival. Assessment in a larger cohort is required to determine utility of these metrics.

\section{MYOCARDIAL EXTRACELLULAR VOLUME IN PATIENTS WITH AORTIC STENOSIS UNDERGOING VALVE INTERVENTION: A MULTICENTRE T1 MAPPING STUDY}

${ }^{1}$ Russell J Everett, ${ }^{2}$ Thomas A Treibel, ${ }^{3}$ Miho Fukui, ${ }^{4}$ Heesun Lee, ${ }^{5}$ Marzia Rigolli, ${ }^{6}$ Anvesha Singh, ${ }^{7}$ Petra Bijsterveld, ${ }^{8}$ Lionel Tastet, ${ }^{7}$ Tarique Al Musa, ${ }^{7}$ Laura Dobson, ${ }^{9}$ Calvin Chin, ${ }^{2}$ Gabriella Captur, ${ }^{10}$ Stephanie Funk, ${ }^{10}$ Jeanette Schulz-Menger, ${ }^{3}$ Erik B Schelbert, ${ }^{8}$ Marie-Annick Clavel, 'David E Newby, ${ }^{5}$ Saul Myerson, ${ }^{8}$ Phillipe Pibarot, ${ }^{3}$ João L Cavalcante, ${ }^{4}$ Seung-Pyo Lee, ${ }^{6}$ Gerry McCann, ${ }^{7}$ John P Greenwood, ${ }^{2}$ James Moon, ${ }^{1}$ Marc R Dweck. 'Centre for Cardiovascular Sciences, University of Edinburgh, Chancellors Building, 49 Little France Crescent, Edinburgh, UK; ${ }^{2}$ Barts Health NHS Trust and University College London, London, UK; ${ }^{3}$ UPMC Cardiovascular Magnetic Resonance Center, Heart and Vascular Institute, Pittsburgh, Pennsylvania, USA; ${ }^{4}$ Cardiovascular Center, Seoul National University Hospital, Seoul, Republic of Korea; ${ }^{5}$ University of Oxford Centre for Clinical Magnetic Resonance Research, Oxford, UK; ${ }^{6}$ Department of Cardiovascular Sciences, University of Leicester and the NIHR Leicester Biomedical Research Centre, Glenfield Hospital, Leicester, UK; ${ }^{7}$ Multidisciplinary Cardiovascular Research Centre and The Division of Biomedical Imaging, Leeds Institute for Cardiovascular and Metabolic Medicine, University of Leeds, Leeds, UK; ${ }^{8}$ Quebec Heart and Lung Institute, Department of Medicine, Quebec, Canada; ${ }^{9}$ National Heart Center, Singapore; ${ }^{10} \mathrm{HELIOS}$ Hospital Berlin-Buch, Berlin, Germany

\subsection{6/heartjnl-2019-BSCMR.16}

Background Diffuse myocardial fibrosis is a key decompensation mechanism in advanced aortic stenosis (AS) and can be quantified using CMR T1 mapping techniques.

Purpose To assess T1 mapping measures of fibrosis in patients with severe AS referred for aortic valve replacement, and determine their associations with clinical characteristics, disease severity and clinical outcome.

Methods In this international prospective cohort study, patients with severe AS underwent CMR at $1.5 \mathrm{~T}$ and $3 \mathrm{~T}$ (Siemens/Philips) with T1 mapping prior to AVR. Image analysis was performed (CVI42, Circle) by a single core laboratory for three T1 mapping measures (native T1, extracellular volume fraction [ECV\%] and indexed extracellular volume $[\mathrm{iECV}=\mathrm{LVMi} * \mathrm{ECV} \%]$ ).

Results Four-hundred patients (70 10 years, $60 \%$ male) from nine international centres (Canada/Germany/Korea/USA/UK) were enrolled (including 144 patients from BSCMR AS700 study). AVR was performed (SAVR: $n=342$, TAVI: $n=58$ ) 19 [4-61] days following CMR, with median of 3.8 [1.7-4.5] years follow-up and 40 deaths recorded. 